\title{
Multiuser Receiver Scheme with SIC for PUCCH in High Speed Train Environment
}

\author{
Wei Wang, Guangliang Ren \\ The State Key Laboratory of ISN, Xidian University, Xi’an, China \\ Email: weiwang1013@gmail.com
}

Received July, 2013

\begin{abstract}
A multiuser receiver scheme with successive interference cancellation (SIC) is proposed to suppress multiuser interference for physical uplink control channel (PUCCH) in high speed train (HST) environment. In the proposed algorithm, each user's signal is detected iteratively in a descending order according to the signal strength at eNB. During each iteration, the strongest signal of all users' is detected and regenerated, and then is subtracted from the composite signal before decoding the next user. Simulation results show that the proposed scheme obtains remarkable gains, e.g. $2 \mathrm{~dB}$ for PUCCH format 2 with 3 users in HST scenario 1. The improvement is more pronounced in the case of increasing number of users, e.g. $3.3 \mathrm{~dB}$ with 6 users.
\end{abstract}

Keywords: PUCCH; Successive Interference Cancellation; High Speed Train; Multiuser

\section{Introduction}

PUCCH is an important narrow-band channel, which carries the uplink control information, such as acknowledge/non-acknowledge (ACK/NACK) bits, downlink channel quality indicator (CQI), scheduling request, precoding matrix indicator (PMI), rank indication (RI) and HARQ-ACK feedback information, and it requires much higher transmission quality than the other channels. With the aid of orthogonal CDMA [1], multiple users share the same time-frequency resources, i.e., a resource block (RB) in the same sub-frame duration in the PUCCH, and the multiuser interference has been the main factor that limits the transmission quality of the control information.

Recently, several literatures have been focused on the scheme of the PUCCH multiuser receiver in the low mobility scenario. Literature [2] proposed a parallel-receiver scheme for the PUCCH multiuser receiver with low reference signals (RSs) density. By using the maximum likelihood (ML) mechanism, literature [3] presented a semi-blind receiver with a high performance. However, the two schemes will be greatly degraded in the HST scenario, because there exists a large Doppler shift, which leads to loss of code orthogonality and also introduces inter-carrier interference (ICI) within a single SCFDMA symbol [4]. Both the two schemes above can not work with so large a Doppler shift without carrier fre-

*This work was supported in part by the State Natural Science Foundation of China, Grant No.61072102 and National Major Specialized Project of Science and Technology, Grant No.2011ZX03001-007-01. quency offset (CFO) estimation and correction. Besides, the near far resistance is high in HST environment due to the fast variation of users' location. The received signal strength at eNB from near users is higher than that from the far ones, and users' power differs greatly, which makes the multiuser interference severe. In this case, the performance of the parallel-receiver is degraded due to the poor performance of the farther users. And it is limited by the number of users while the user density in HST is very high. Semi-blind algorithm has higher computational complexity with increasing number of users and CQI bits. Consequently, the available receiver schemes can not directly apply to such a special scenario. A receiver, which can overcome the influence of frequency offset and power differences among users, is required to maintain the link quality for PUCCH in HST environment. Hence, we propose a multiuser receiver scheme with SIC for PUCCH. The proposed scheme can greatly suppress the interference of the strong on the weak and is robust to the number of users, increasing the system capacity. Moreover, it has lower complexity and is suitable for implementation.

The following sections are organized as: In section 2, system model is described. In section 3 , the multiuser receiver scheme with SIC is proposed and in section 4 simulation results are presented and analyzed. Finally, basic conclusion is drawn in section 5 .

\section{System Model}

A PUCCH subframe consists of two $0.5 \mathrm{~ms}$ slots, each of 
which contains seven SC-FDMA symbols and occupies 12 subcarriers distributing at band edges. PUCCH supports seven formats, namely formats $1 / 1 \mathrm{a} / 1 \mathrm{~b}$, format $2 / 2 \mathrm{a} / 2 \mathrm{~b}$ and format 3 . The receiver we proposed applies to all PUCCH formats. In the following, we mainly talk about format 2 for the transmission of CQI bits. The time frequency structure of PUCCH format 2 is shown in Figure 1. CQI information bits from different users are coded into 20 bits by a RM code [5], and then these bits are QPSK modulated, resulting in a block of complex-valued modulation symbols $d_{0}, d_{1}, \cdots, d_{9}$. Those data symbols occupy 10 SC-FDMA symbols of a subframe. The rest symbols of a PUCCH format 2 subframe are used to transmit RSs. Each symbol shall be spreaded by a cyclically shifted length 12 sequence. The sequence of different users in a cell, generated by phase-rotated basic sequence, is orthogonal to each other. The rotation value of user $\mathrm{k}$ can be expressed as $\alpha=\alpha_{1}+\alpha_{k}$, where $\alpha_{l}$ varies with symbols and $\alpha_{k}$ varies with users [6]. The $k$ th user's modulated symbols in a subframe, spreading sequence of the lth symbol and spreaded symbol $l$ can be described respectively as

$$
\begin{aligned}
& \boldsymbol{s}_{k}= {\left[s_{k, 0}, s_{k, 1}, \cdots, s_{k, 13}\right] } \\
&= {\left[\begin{array}{l}
d_{k, 0}, 1, d_{k, 1}, d_{k, 2}, d_{k, 3}, 1, d_{k, 4}, \\
d_{k, 5}, 1, d_{k, 6}, d_{k, 7}, d_{k, 8}, 1, d_{k, 9}
\end{array}\right] } \\
& \boldsymbol{c}_{k, l}= {\left[c_{k, l}(0), c_{k, l}(1), \cdots, c_{k, l}\left(N_{S C}^{R B}-1\right)\right]^{T} } \\
& \bar{s}_{k, l}=s_{k, l} \cdot c_{k, l}
\end{aligned}
$$

where $k=0,1 \cdots, K-1$. $K$ is the total number of users. $l$ denotes symbol index within a subframe and $l=0,1, \cdots, 13 . \quad i=0,1, \cdots, N_{S C}^{R B}-1 . \quad N_{S C}^{R B}$ is the number of subcarriers in a RB. $\overline{\boldsymbol{s}}_{k, l}$ is mapped to a group of localized subcarriers allocated for the kth user. By using the N-point inverse fast Fourier transform, an SC-FDMA symbol of the $k$ th user can be obtained as follows

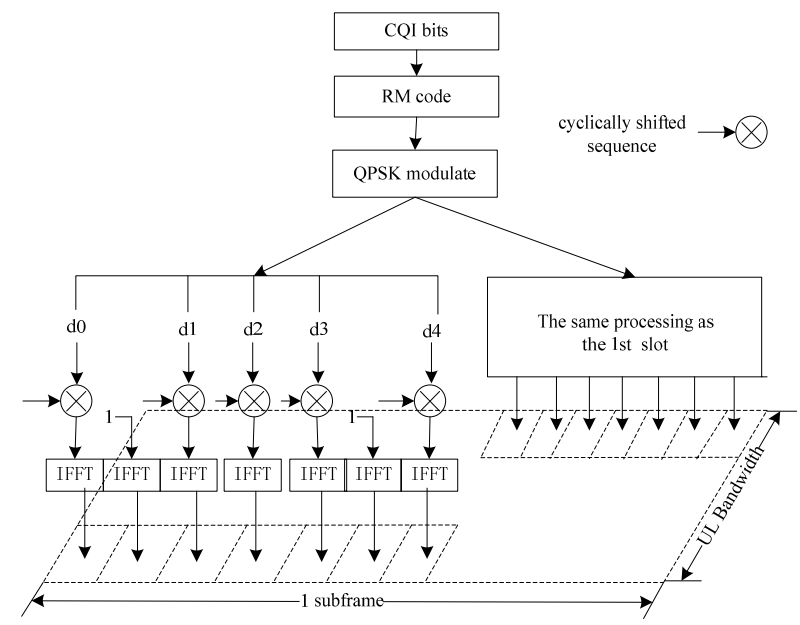

Figure 1. Time-frequency structure of PUCCH format 2.

$$
\begin{aligned}
x_{k, l}(n) & =\frac{1}{N} \sum_{m=0}^{N-1} s_{k, l} a_{k, l}(m) \exp \left(j 2 \pi \frac{m n}{N}\right) \\
n & =0,1, \cdots, N-1
\end{aligned}
$$

where $\boldsymbol{a}_{k, l}$ is mapped by the length-12 sequence $\boldsymbol{c}_{k, l}$, and $\boldsymbol{a}_{k, l}=\left[a_{k, l}(0), \cdots, a_{k, l}(N-1)\right]$.

In HST channel model, the LOS component is constant and frequency offset exists caused by the Doppler shift. The scattered component in the uplink also has frequency offset half that of LOS component [4]. The received complex baseband symbol in time domain can be described as

$$
\begin{aligned}
r_{l}(n)= & \sum_{k=0}^{K-1} x_{k, l}(n)\left[\exp \left(j 2 \pi \varepsilon_{k} \frac{N_{g}+l N_{s}+n}{N}\right) c+\right. \\
& \left.\exp \left(j \pi \varepsilon_{k} \frac{N_{g}+l N_{s}+n}{N}\right) h_{k, l}^{s}(n)\right]+w_{l}(n)
\end{aligned}
$$

where $N_{g}$ is the length of the cyclic prefix (CP). $N_{s}$ indicates the samples in a SC-FDMA symbol, and $N_{s}=N_{g}+N . \quad c$ and $h_{k, l}^{s}(n)$ represent the coefficient of LOS component and NLOS component respectively. $\varepsilon_{k}$ is normalized CFO of the LOS component. $w_{l}(n)$ is the AWGN term.

By removing CP, FFT process and subcarrier demapping at eNB, the received signal on the $m t h$ subcarrier during the lth SC-FDMA symbol can be represented as

$$
\begin{aligned}
R_{l}(m)= & \sum_{n=0}^{N-1} r_{l}(n) \exp \left(-\frac{j 2 \pi n m}{N}\right) \\
= & \exp \left(j 2 \pi \varepsilon_{k} \frac{N_{g}+l N_{s}}{N}\right) s_{k, l} a_{k, l}(m) H_{k, l} \\
& +I_{k, l}(m)+U_{k, l}(m)+W_{l}(m) \\
H_{k, l}=\frac{1}{N} \sum_{n=0}^{N-1}\left[c+\exp \left(-j \pi \varepsilon_{k} \frac{N_{g}+l N_{s}+n}{N}\right) h_{k, l}^{s}(n)\right] & \cdot \exp \left(j 2 \pi \frac{n}{N} \varepsilon_{k}\right)
\end{aligned}
$$

where $I_{k, l}(m)$ denotes the ICI of the $k t h$ user. $U_{k, l}(m)$ is the interference of the other users to the $k t h$ user and $W_{l}(m)$ is noise in frequency domain. $H_{k, l}$ is an attenuation coefficient caused by channel and residual carrier frequency offset.

\section{Proposed Scheme}

In this section, the proposed SIC-receiver is described in detail. In the scheme, the symbol of each user is detected in a descending order based on the received power levels. The strongest signal of all users' in each iteration is detected and regenerated, and then the contribution of the strongest signal is cancelled from the composite signal 
before the detection of the next user, from which the multiuser interference is progressively reduced. Symbol detection of the each user is performed by single user detection (SUD) method. Firstly, some of the SUD combining methods considered in this paper are reported.

$H_{k, l}$ is estimated by the scheme proposed in [2], and the carrier frequency offset is estimated by a iteration method in [7]. Let $\hat{H}_{k, l}$ denote the estimation of $H_{k, l}$, and $\hat{\varepsilon}_{k}$ the estimation of $\varepsilon_{k}$.

Define $v$ as the iteration number. Initialize $R_{l}^{0}(i)$ to $R_{l}(i)$. The SIC algorithm for PUCCH in HST scenario is described as follows.

Step 1, despreading and power calculation. The lth symbol of user $k$ is expressed as

$$
\bar{R}_{k, l}^{v}=\frac{\boldsymbol{c}_{k, l}^{H} \cdot \boldsymbol{R}_{l}^{\nu-1}}{N_{S C}^{R B}}
$$

where $\quad \boldsymbol{R}_{l}^{\nu-1}=\left[R_{l}^{\nu-1}\left(m_{0}\right), R_{l}^{\nu-1}\left(m_{1}\right), \cdots, R_{l}^{\nu-1}\left(m_{11}\right)\right]^{T} . \quad m_{i}$ is the useful subcarrier index.

Let $p_{k}^{v}$ be the power of user $k$ in the $v$ th iteration, find the user of max-power, and let

$$
\kappa=\underset{k}{\arg \max }\left\{P_{1}^{v}, P_{2}^{v}, \cdots, P_{K}^{v}\right\} .
$$

Step 2, data symbol detection of the $\kappa$ th s user.

$$
\hat{s}_{\kappa, l}^{v}=Q\left\{\exp \left(-j 2 \pi \hat{\varepsilon}_{\kappa}\right) \hat{H}_{\kappa, l}^{*} \bar{R}_{\kappa, l}\right\}
$$

where $Q\{\cdot\}$ is a quantization operation, and it assigns an element of the data symbol alphabet to each value of $\exp \left(-j 2 \pi \hat{\varepsilon}_{\kappa}\right) \hat{H}_{\kappa, l}^{*} \bar{R}_{\kappa, l}$.

Step 3, signal regeneration. The lth SC-FDMA symbol of the $\kappa$ th user is regenerated as

$$
\begin{gathered}
\hat{\bar{r}}_{\kappa, l}^{v}(n)=\frac{1}{N} \sum_{m=0}^{N-1} \hat{s}_{\kappa, l}^{v} a_{\kappa, l}(m) \hat{H}_{\kappa, l} \exp \left(j 2 \pi \frac{m n}{N}\right) \\
\cdot \exp \left(j 2 \pi \hat{\varepsilon}_{\kappa} \frac{N_{g}+l N_{s}+n}{N}\right)
\end{gathered}
$$

Step 4, subtraction of the regenerated max-power signal. Let $\hat{\bar{R}}_{\kappa, l}^{v}(i)$ be the transformation of $\hat{\bar{r}}_{\kappa, l}^{v}(n)$ in frequency domain. Subtract $\bar{R}_{\kappa, l}^{v}(i)$ from the composite signal, and we have

$$
R_{l}^{v}(i)=R_{l}^{v-1}(i)-\hat{\bar{R}}_{\kappa, l}^{v}(i)
$$

Step 5, the power of user $\kappa$ is calculated again and is denoted as $\bar{P}_{\kappa}^{v}$. If $\bar{P}_{\kappa}^{v}<P_{\kappa}^{v}$, the next iteration follows on. Otherwise, set $R_{l}^{v}(i)=R_{l}^{v-1}(i)$, and the power of the $\kappa t h$ user zero in the following iteration. Then start the next iteration. The iteration will not stop until the weakest user is detected. Moreover, the data symbols detection of a user may be performed repeatedly due to the iteration process. The one when the user's power is maximum is chosen as the final result.

\section{Simulation Results}

In this section, the performances of the proposed receiver scheme and the parallel-receiver scheme are investigated. The simulation parameters are listed in Table $\mathbf{1}$.

Block error ratio (BLER) of CQI in HST scenario 1 and 2 is shown. Consider a worse case that the SNR of the strong is higher than that of the weak by $9.5 \mathrm{~dB}$, and when the number of users is three, one has higher power; the number is 2 while there are 6 users. The noise attenuation varies with the average power of all users' to keep SNR constant. The simulation results are presented as follows.

Figure 2 presents the average BLER of SIC-receiver and parallel-receiver with $8 \mathrm{CQI}$ bits for PUCCH format 2 in HST scenario 1 . The proposed algorithm provides a gain of around $2 \mathrm{~dB}$ and $3.3 \mathrm{~dB}$ over the parallel-receiver for 3 users and 6 users respectively. The proposed scheme is robust to number of users, whereas the performance of parallel-receiver decreases with the increasing number of users.

Table 1. Simulation parameters.

\begin{tabular}{ll}
\hline \multicolumn{1}{c}{ Parameters } & \multicolumn{1}{c}{ Description } \\
\hline System bandwidth & $10 \mathrm{M}$ at $2.3 \mathrm{GHz}$ \\
Number of FFT/IFFT points & 1024 \\
PUCCH formats & Format 2 \\
Channel model & HST \\
\# Users & 3 Users, 6 Users \\
$\alpha$ shifts & {$[0,1,2],[0,1,2,3,4,5]$} \\
Velocity of the train & $350 \mathrm{~km} / \mathrm{h}$ \\
\hline
\end{tabular}

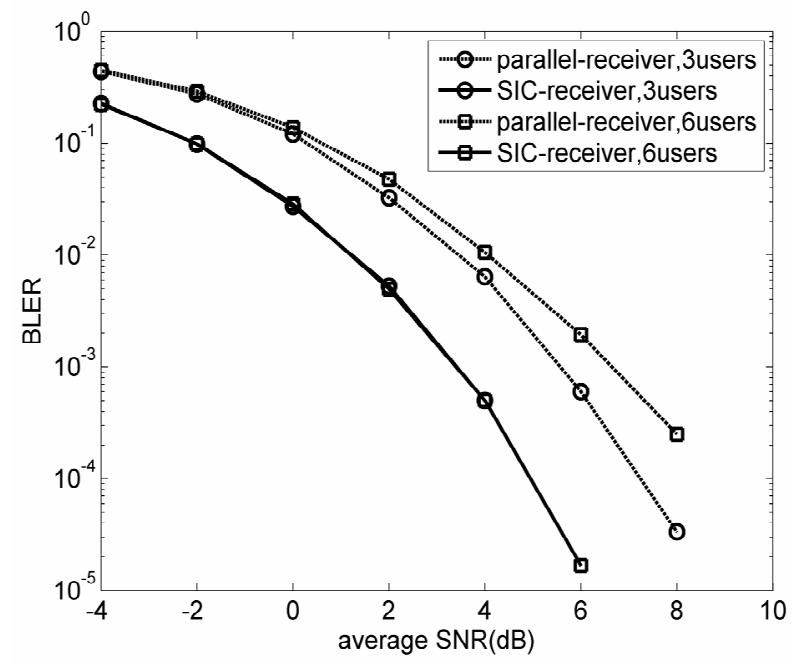

Figure 2. SIC-receiver vs. parallel -receiver, scenario 1. 


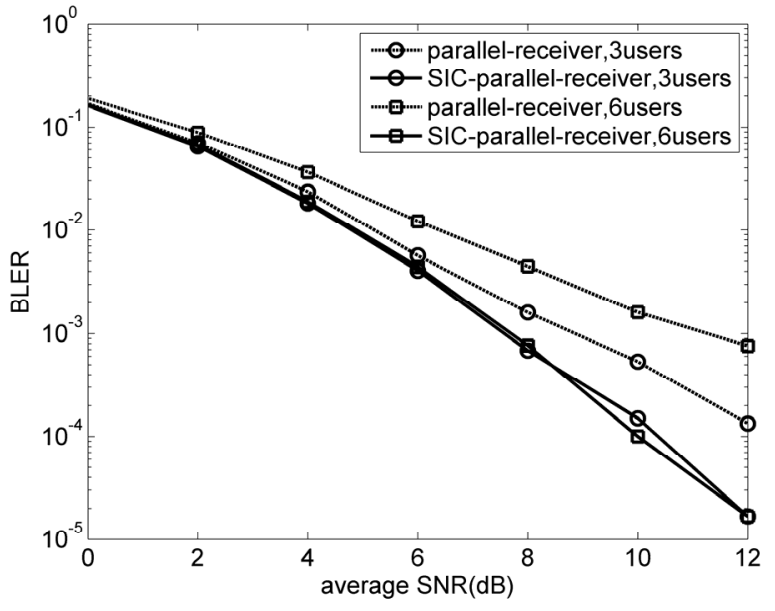

Figure 3. SIC-parallel-receiver vs. parallel-receiver, scenario 2.

We consider a SIC-parallel-receiver in HST scenario 2. BLER of CQI of the SIC-parallel-receiver comparing with the parallel-receiver is shown in Figure 3. The parallel-receiver with SIC performs much better over the parallel-receiver, providing a gain of around $1.3 \mathrm{~dB}$ and $3.4 \mathrm{~dB}$ when BLER achieves $10^{-3}$ for 3 users and 6 users respectively. The improvement is more pronounced with 6 users. It is clear that the gaps tend to be more significant for higher signal to noise ratio (SNR) values. This is because the limiting factor of performance at lower SNR is noise, whereas it is multiuser interference at higher SNR. The parallel-receiver with SIC in HST scenario 2 is also robust to the number of users.

\section{Conclusions}

In this paper, a multiuser receiver scheme with SIC for PUCCH in LTE system is discussed. An iteration method is adopted to reduce multiuser interference. During each iteration, the max-power user is detected, regenerated and then subtracted from the composite signal before the detection and cancellation of the next user. Simulation results show that it improves the BLER performance for
PUCCH receiving. The improvement is more pronounced with increasing number of users. The proposed system is robust to multiuser interference and near-far resistance, which translates to significant increase in system capacity.

\section{REFERENCES}

[1] 3rd Generation Partnership Project (3GPP), "Evolved Universal Terrestrial Radio Access (E-UTRA); Physical Channels and Modulation (Rel-11)," Available: http://www.3gpp.org/ftp/specs/archive/36_series/36.211/. htm, Mar. 2013.

[2] D. Wang, S. Z. Yang, Y. Liao and Y. Liu, "Efficient Receiver Scheme for LTE PUCCH,” IEEE Communications Letters, Vol. 16, 2012, pp. 352-355. doi:10.1109/LCOMM.2012.012412.112251

[3] H. Yang, D. Astely, R. Baldemair and S. Falahati, "Semi-blind Multi-user Detection for LTE PUCCH," Proceedings of Wireless Communications and Networking Conference, Budapest, Hungary, 2009, pp. 1-5.

[4] L. H. Yang, G. L. Ren, B. K. Yang and Z. L. Qiu, "Fast Time-Varying Channel Estimation Technique for LTE Uplink in HST Environment,” IEEE Transactions on Vehicular Technology, Vol. 61, No. 9, 2012, pp. 4009-4019. doi:10.1109/TVT.2012.2214409

[5] 3rd Generation Partnership Project (3GPP), "Evolved Universal Terrestrial Radio Access (E-UTRA); Multiplexing and channel coding (Rel-11)." Available: http://www.3gpp.org/ftp/specs/archive/36_series/36.212/. htm, Feb. 2013.

[6] M. Zhou, B. Jiang, W. Zhong and X. Gao, "Efficient Channel Estimation for LTE Uplink," Proceedings of Wireless Communications \& Signal Processing Conference, Nanjing, China, 2009, pp. 1-5.

[7] X. N. Ren, G. L. Ren and C. H. Le, "Carrier Frequency Offset Estimation for PUCCH in High Speed Train Environment," Proceedings of the 12th International Conference on Electronic Packaging Technology and High Density Packaging, Shanghai, China, 2011, pp. 1-4. 